\title{
Workshop on advanced polarimetric instrumentation: API'09
}

\author{
E. Garcia-Caurel
}

LPICM, Ecole Polytechnique, CNRS, 91128, Palaiseau, France

\begin{abstract}
Preface of the workshop The First NanoCharM Workshop on Advanced Polarimetric Instrumentation was focused on the instrumentation developed to work with polarised light, which is the cause of a great deal of research and study in a variety of fields, such as astronomy, telecommunications, defence, medicine, electronics, material science, metrology and many more. The workshop was therefore organised around six themes: imaging, astronomy and satellites, measurement of optical activity, Mueller matrix algebra and polarimetric data analysis, diffractometry and metrology of nanostructures, as well as new developments in polarimetric instrumentation. The workshop was founded by the European project NanoCharM the aim of which is, firstly, to coordinate research activities focusing on the characterisation of materials using polarised light (polarimetry) and, secondly, to develop communication initiatives to make scientists, decision-makers in the broadest sense of the term, industrialists and students aware of the various applications of polarised light.
\end{abstract}

